\title{
Entwicklung von ökotoxikologischen Instrumenten und ihre rechtliche Implementierung zur marinen ökologischen Risikobewertung von Chemikalien, Pestiziden und Baggergut
}

\author{
Carolin Floeter $\cdot$ Hans Toni Ratte $\cdot$ Wolfgang Ahlf
}

Erhalten: 19. September 2008/Akzeptiert: 24. September 2008/Online veröffentlicht: 29. Oktober 2008

(C) Springer-Verlag 2008

Zusammenfassung Hintergrund und Ziel Die marine Umwelt ist oftmals die finale Senke für Schadstoffe, insbesondere für persistente, bioakkumulative und toxische Schadstoffe (PBT Substanzen). Die Schadstoff-Belastung von marinen Top-Prädatoren ist in der Nord- und Ostsee und aufgrund des Schadstoffferntransportes sogar auch in der Arktis besorgniserregend.

Folglich war zu prüfen, ob das europäische Umweltrecht bei der prospektiven Risikobewertung von Chemikalien, Pestiziden und Baggergut auch die marine Umwelt in Form einer gesonderten marinen ökologischen Risikobewertung berücksichtigt.

Resultate Die juristische Analyse des europäischen Umweltrechts zeigte, dass die europäischen Schutzzielbestim-

Verantwortlicher Herausgeber: Henner Hollert

Die Dissertation wurde im Januar 2006 zur Begutachtung an der TUHH eingereicht und 2007 im Shaker Verlag veröffentlicht. Aufgrund der sehr dynamischen Entwicklungen im EU Umweltrecht in den Jahren 2006-2008 wurden ergänzende Aktualisierungen des EUumweltrechtlichen Teils dieser Arbeit für diese Veröffentlichung vorgenommen. Die aktuellen Rechtsentwicklungen haben das Ergebnis der abgeschlossenen Rechtsanalyse nicht grundlegend verändert.

C. Floeter, geb. Peters $(\bowtie)$

Science and Law Consult,

Woyrschweg 39, 22761 Hamburg, Deutschland

E-Mail: carolin.floeter@science-and-law.de

H. T. Ratte

Rheinisch-Westfälische Technische Hochschule Aachen,

Institut für Umweltforschung (Biologie V),

Lehrstuhl für Umweltbiologie und -chemodynamik,

Worringerweg 1, 52056 Aachen, Deutschland

\section{W. Ahlf}

Technische Universität Hamburg-Harburg, Institut für Umwelttechnik und Energiewirtschaft, Eissendorfer Straße 40, 21073 Hamburg, Deutschland mungen eine gute Qualität von Küstengewässern bis 2015 (EU-Wasserrahmenrichtlinie) und einen guten Qualitätszustand der europäischen Meere bis 2020 (MeeresstrategieRahmenrichtlinie) vorsehen, aber eine prospektive ökologische Risikobewertung von Chemikalien und Pestiziden für Meerwasserökosysteme insgesamt nur unzureichend im europäischen Umweltrecht verankert ist.

Diskussion Es wurde dargelegt, dass eine alleinige Risikobewertung für Süßwasserökosysteme aufgrund der Besonderheiten der Schadstoffwirkung in der marinen Umwelt für einen umfassenden Schutz der Meeresumwelt nicht ausreicht. Eine statistische Auswertung internationaler Datenbanken zur relativen Toxizität von Narkotika gab einen Hinweis darauf, dass marine Organismen gegenüber Substanzen mit einem unspezifischen Wirkmechanismus signifikant sensitiver als ihre Süßwasservertreter sein können. Ca. $60 \%$ der Industriechemikalien werden nach ihrem Mode of Action (MoA) als Narkotika klassifiziert und sind somit für die Umwelt von großer Bedeutung. Aufgrund ihrer hydrophoben Eigenschaften und ihrer geringeren Löslichkeit im Meerwasser war insbesondere die ökotoxikologische Risikobewertung von marinen Sedimenten von Bedeutung. Es wurde ein marines Biotest-Set aufgebaut und weiterentwickelt, um das ökotoxikologische Potenzial von Schadstoffen in aestuarinen und marinen Sedimenten zu erfassen. Ein Test-Set bestehend aus drei Biotestverfahren wurde auf die Besonderheiten unter Meerwasserbedingungen angepasst, sowie untereinander und im internationalen Vergleich harmonisiert und validiert.

Schlussfolgerungen Neben zwei Testverfahren für die Bewertung des wässrigen Eluats mariner Sedimente (Leuchtbakterientest und mariner Algentest) war vor allem auch die Etablierung und Weiterentwicklung eines Gesamtsedimenttests mit dem Amphipoden Corophium volutator für die Risikobewertung von aestuarinen und marinen Sedimenten 
wichtig. Um einen das ganze Jahr verfügbaren, standardisierteren Testorganismus zu erhalten, wurde Corophium volutator erstmals zu allen Jahreszeiten unter Laborbedingungen zur Reproduktion gebracht. Die Reproduktionsergebnisse stellen eine essenzielle Basis für den international dringend geforderten chronischen Gesamtsedimenttest dar. Die Forschungsergebnisse sind direkt in die internationale (ISO), europäische (EN) und nationale (DIN) Standardisierung gemündet, so dass jetzt drei standardisierte und validierte Testverfahren für die Bewertung mariner Sedimente und zur Risikobewertung von Chemikalien und Pestiziden zur Verfügung stehen.

Empfehlungen und Perspektiven Es wurde ferner aufgezeigt, wie eine marine ökologische Risikobewertung im europäischen Umweltrecht vollzogen werden müsste, um den international formulierten Schutzzielbestimmungen eines nachhaltigen Meeresumweltschutzes gerecht zu werden.

Schlüsselwörter Baggergut · Chemikalien · Corophium volutator (Amphipode) · Entwicklung von ökotoxikologischen Instrumenten · EU Wasserrahmenrichtlinie · Europäisches Umweltrecht · Gesamtsedimenttest · Leuchtbakterientest - Marine ökologische Risikobewertung · Marine Sedimente $\cdot$ Mariner Algentest $\cdot$ MeeresstrategieRahmenrichtlinie $\cdot$ Narkotika $\cdot$ Pestizide $\cdot \mathrm{REACH}$

\section{Development of ecotoxicological instruments and their legal implementation for the marine ecological risk assessment of chemicals, pesticides and dredged material}

\begin{abstract}
Background, aim, and scope The marine environment is often the final sink for pollutants, especially for persistent organic pollutants (POPs) and for persistent, bioaccumulative and toxic (PBT) substances. The body burden of marine top predators in the Baltic Sea, in the North Sea and also in the Arctic seas is alarming.

Thus, the question was investigated if the European environmental law considers a prospective marine ecological risk assessment of chemicals, pesticides and dredged material before they are launched on the market or disposed to the sea.
\end{abstract}

Results The analysis of European environmental law showed that the European environmental protection goals demand a good quality status of coastal waters until 2015 (Waterframework Directive) and a good quality status of European seas until 2020 (Marine Strategy Directive), but a specific marine ecological risk assessment of chemicals and pesticides is not sufficiently required in current European legislation.

Discussion It was shown that the ecological risk assessment for freshwater ecosystems is, due to the pecularities of pollutant impacts in the marine environment, not adequate to predict marine effects and to protect the marine environment sufficiently. A statistical analysis of international databases on the relative toxicity of narcotics revealed that marine organisms can be significantly more sensitive than freshwater organisms towards substances with an unspecific mode of action (narcotics). Approximately $60 \%$ of the industrial chemicals are classified as narcotics by their mode of action. Thus, this substance class is of environmental importance. Due to the hydrophobic properties and the low solubility of narcotics in seawater, the ecotoxicological assessment of marine sediments was of interest. An estuarine and marine bioassay test set was established and further developed to assess the ecotoxicological potential of brackish and seawater sediments. It was important that the test procedures were adapted to brackish and marine conditions and were harmonised between each other as well as on the international level.

Conclusions Beside two bioassays for the ecotoxicological assessment of elutriates of marine and brackish sediments (bacteria bioluminescence test and marine algae test), the implementation and further development of the whole sediment bioassay with the marine amphipod Corophium volutator was important for enhancing the risk assessment. In order to gain a more standardised, all-season available test organism, the marine amphipod was for the first time reproduced under laboratory conditions the whole year round (also in winter), which is the essential basis for the urgently needed chronic whole sediment bioassay. The results of this investigation were implemented in the international (ISO), European (EN) and national (DIN) standardisations. Therefore, a standardised test set is ready for the implementation in the marine ecological risk assessment of chemicals, pesticides and dredged material in international, European and national legislation.

Recommendations and perspectives Recommendations to improve the implementation of a marine risk assessment in European regulations are given with the goal to reach the internationally required objective of a sustainable development of the seas.

Keywords Bacteria bioluminescence test Chemicals . Corophium volutator (amphipod) $\cdot$ Development of ecotoxicological instruments · Dredged material · European environmental law $\cdot$ Marine algae test $\cdot$ Marine ecological risk assessment $\cdot$ Marine sediments $\cdot$ Marine Strategy Directive $\cdot$ Narcotics $\cdot$ Pesticides $\cdot$ REACH $\cdot$ Waterframework Directive $\cdot$ Whole-sediment bioassay

\section{Einleitung}

Die Meeresumwelt ist vielfachen Belastungen ausgesetzt. Sowohl in der Nordsee als auch in der Ostsee ist ein Rück- 
Berücksichtigung der marinen ökologischen Risikobewertung im europäischen Umweltrecht ?

$+\rightarrow$

Besteht die Notwendigkeit einer gesonderten marinen ökologischen Risikobewertung?

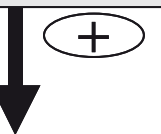

Weiterentwicklung u. Standardisierung eines minimalen Biotest-Sets für Brackwasser- und Meerwasser-Sedimente

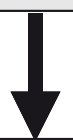

Entwicklungen für einen aestuaren und marinen chronischen Gesamtsedimenttest

Empfehlungen für die Implementierung der marinen ökologischen Risikobewertung ins europäische Umweltrecht

Abb. 1 Schematische Übersicht über die Struktur der Arbeit (Floeter 2007)

gang der Biodiversität zu verzeichnen und zahlreiche Arten sind in ihrem Vorkommen bedroht (SRU 2004). Auch wenn die Schadstoffbelastung der marinen Ökosysteme nur ein Faktor unter mehreren Stressoren ist, ist deren Bedeutung nicht zu unterschätzen, denn die marine Umwelt ist für viele Schadstoffe die ultimative Senke (O'Shea et al. 2003). Die meisten marinen Säugetiere sind Carnivore und stehen als Top-Prädatoren am Ende der Nahrungskette (Moeller 2003). Das Gesundheitsrisiko dieser Tiere durch die Exposition gegenüber Xenobiotika ist aufgrund der Bioakkumulation dieser Substanzen möglicherweise sehr groß, da marine Säugetiere einen hohen Fettgehalt aufweisen und viele Xenobiotika lipophile Eigenschaften haben (Moeller 2003). Marine Säugetiere können beträchtliche Schadstoffkonzentrationen sowohl über die Muttermilch als auch über die Nahrungskette (Biomagnifikation) akkumulieren (Gregory und Cyr 2003). Die Tatsache, dass hohe Konzentrationen zum Teil auch noch für Stoffe gemessen werden, deren Verwendung bereits seit Jahren eingeschränkt oder sogar verboten ist (z.B. PCB, DDT und Lindan), verdeutlicht das besondere Risikopotenzial von persistenten organischen Schadstoffen (POPs) und von persistenten, bioakkumulativen und toxischen (PBT) Substanzen. Gelangen diese Substanzen erst einmal in die Um- welt, so ist ihre Verbreitung aufgrund des atmosphärischen Schadstoffferntransports global und nicht reversibel. Persistente organische Chemikalien (wie z.B. PCBs, PCDD, PCDFs, DDT) und chlorierte Benzene (z. B. HCB) werden nicht nur in marinen Säugetieren der Nordsee und Ostsee sondern auch in der Arktik nachgewiesen (O'Hara und Becker 2003). Neben den alten POPs, deren Wirkung in der marinen Umwelt nach langjähriger Exposition bekannt ist, werden kontinuierlich Substanzen der zweiten und dritten Generation als Problemstoffe mit PBT Eigenschaften identifiziert. Zu ihnen zählen z.B. synthetische Moschusverbindungen (Tanabe 2005), polybromierte Diphenylether (PBDEs) (Tanabe 2004) und perfluorierte organische Substanzen (PFOCs, PFOS, PFOA) (Ebinghaus und Temme 2006). Zwischen der Erkenntnis über die Gefährlichkeit einer Substanz, der Identifikation der weltweiten Verbreitung und des Nachweises von Effekten in marinen Ökosystemen können Jahrzehnte vergehen.

Ziel dieser Arbeit war, die prospektive marine ökologische Risikobewertung von Chemikalien, Pestiziden und Baggergut im europäischen Umweltrecht zu überprüfen, ökotoxikologisch weiterzuentwickeln und für eine Implementierung im europäischen Umweltrecht bereitzustellen (Abb. 1). 


\section{Europäisches Umweltrecht}

\subsection{Schutzziele für die marine Umwelt} nach europäischem Umweltrecht

EU-Wasserrahmenrichtlinie Ziel der EU-Wasserrahmenrichtlinie (WRRL, EG 2000) ist es, für alle Oberflächengewässer einen guten Zustand und für alle künstlichen oder erheblich veränderten Wasserkörper ein gutes ökologisches Potenzial und einen guten chemischen Zustand bis zum Jahr 2015 zu erreichen (Art. 4 ii, iii). Für Küstengewässer wird der gute Zustand durch biologische, hydromorphologische und physikalisch-chemische Qualitätskomponenten bestimmt. Hinsichtlich der Schadstoffbelastung ist ein guter Zustand im Küstengewässer erreicht, wenn die synthetischen und spezifischen nicht-synthetischen Schadstoffe in Konzentrationen vorkommen, die nicht höher als die Umweltqualitätsnormen sind (Anhang V 1.2.4).

Blaubuch „Integrierte Meerespolitik für die Europäische Union " Mit dem Blaubuch (EC 2007) wurde von der Europäischen Kommission ein europäisches Konzept zur wirtschaftlichen Entwicklung und Nutzung der Küsten- und Meeresräume vorgestellt. Es wird die Bedeutung der Meere als Europas „Lebensblut“ hervorgehoben. Es sei ein ,integriertes sektorübergreifendes Konzept für die Meerespolitik erforderlich, um den Herausforderungen der Globalisierung, dem Klimawandel, der Gefährdung der Meeresumwelt, der Energieversorgungssicherheit und der Nachhaltigkeit sowie dem demografischen Druck auf Europas Küsten besser als bisher zu begegnen." Trotz dieser Erkenntnis steht im Blaubuch die wirtschaftliche Nutzung der Meere im Vordergrund, dem Meeresumweltschutz wird wenig Raum geschenkt. Vielmehr wird auf die thematische Meeresschutzstrategie und EU-Meeresstrategie-Rahmenrichtlinie als Umweltsäule der zukünftigen Meerespolitik verwiesen.

Thematische Strategie für den Schutz und die Erhaltung der Meeresumwelt In der thematischen Strategie (EC 2005) wurde der Zustand der Meeresumwelt als besorgniserregend eingestuft: Das Fortschreiten der Verluste an biologischer Vielfalt, der Grad der Verunreinigungen durch gefährliche Stoffe und die Folgen der Klimaveränderungen seien einige der am deutlichsten sichtbaren Warnsignale (EC 2005). Es wurde das Gesamtziel formuliert, die nachhaltige Nutzung der Meere zu fördern und Meeresökosysteme zu erhalten. Ein hohes Schutzniveau sei eine unabdingbare Voraussetzung für die Nutzung des vollen wirtschaftlichen Potenzials der Ozeane und Meere. Es wurde die Notwendigkeit gesehen, dass ein integriertes Konzept entwickelt werden müsse, bei dem alle Arten von Belastungen berücksichtigt und klare, operationelle Ziele und Maßnahmen festgelegt werden, um weitere Verluste an biologischer Vielfalt und eine Verschlechterung der Meeresumwelt zu vermeiden. Jedoch sieht die thematische Strategie kein sektorübergreifendes in- tegratives Konzept zum Meeresumweltschutz vor, sondern gibt die Verantwortung an die Mitgliedstaaten zurück. Die konkreten Zielvorgaben und Maßnahmeprogramme werden weitestgehend den Mitgliedstaaten überlassen. Damit steht die Strategie diametral zu der Erkenntnis, dass gerade für die grenzüberschreitenden Meere länderübergreifende Maßnahmeprogramme entwickelt werden müssen. Es wird lediglich gefordert, dass die Länder einer Meeresregion Bewirtschaftungseinheiten bilden und ihre Maßnahmeprogramme untereinander koordinieren. Es ist mit dem vorgelegten Konzept der Renationalisierung nicht ersichtlich, wie auf EU-Ebene Fortschritte bei der Umsetzung internationaler Übereinkommen zum Schutz der Meeresumwelt, wie OSPARCOM und HELCOM, erzielt werden sollen (SRU 2006). So wird in diesem Entwurf, im Gegensatz zum ersten Entwurf (EC 2002), das völkerrechtlich verankerte Generationsziel zur Reduktion von Schadstoffen (OSPARCOM 1998b) nicht verankert. Mit dem vorliegenden Konzept wurde der dringenden Notwendigkeit einer Weiterentwicklung des europäischen Umweltrechts für einen sektorübergreifenden, integrativen Meeresumweltschutz nicht nachgekommen.

EU-Meeresstrategie-Rahmenrichtlinie Die Konkretisierung der europäischen Meeresschutzstrategie erfolgt durch die EU-Meeresstrategie-Rahmenrichtlinie (EG 2008). Diese soll einen Rahmen schaffen, aufgrund dessen die Mitgliedstaaten die notwendigen Maßnahmen ergreifen, um spätestens bis zum Jahr 2020 einen guten Zustand der Meeresumwelt zu erreichen oder zu erhalten (Art. 1).

Die Richtlinie soll sich auf die Meeresregionen der Mitgliedstaaten erstrecken und umfasst sowohl den Wasserkörper als auch den Meeresboden (Art. 3 I). Der „gute Umweltzustand" wird in der Richtlinie definiert als der Umweltzustand, den Meeresgewässer aufweisen, bei denen es sich um ökologisch vielfältige und dynamische Ozeane und Meere handelt, die im Rahmen ihrer jeweiligen Besonderheiten sauber, gesund und produktiv sind und deren Meeresumwelt auf nachhaltigem Niveau genutzt wird, so dass die Nutzungs- und Betätigungsmöglichkeiten der gegenwärtigen und der zukünftigen Generationen erhalten bleiben. Dies heißt u. a., dass die vom Menschen verursachten Einleitungen von Stoffen in die Meeresumwelt keine Verschmutzungseffekte verursachen (Art. $3 \mathrm{~V}$ ).

Der gute Umweltzustand wird auf der Ebene der jeweiligen Meeresregion anhand qualitativer Deskriptoren (Anhang I) festgelegt. Zu ihnen zählen neben ökologischen auch folgende Deskriptoren: „Aus den Konzentrationen an Schadstoffen ergibt sich keine Verschmutzungswirkung. Schadstoffe in für den menschlichen Verzehr bestimmtem Fisch und anderen Meeresfrüchten überschreiten nicht die im Gemeinschaftsrecht oder in anderen einschlägigen Regelungen festgelegten Konzentrationen. Die Eigenschaften und Mengen der Abfälle im Meer haben keine schädlichen Auswirkungen auf die Küsten- und Meeresumwelt.“ 
Der derzeitige Richtlinienentwurf kann hinsichtlich seiner Zielbestimmung eines guten Umweltzustandes bis zum Jahr 2020 positiv bewertet werden. Es bleibt jedoch kritisch abzuwarten, ob die Mitgliedstaaten dem Anspruch eines hohen Schutzniveaus genügen werden. Das Blaubuch und die Meerestrategie-Rahmenrichtlinie liefern bisher kein integratives Konzept für das Stoffstrommanagement, welches das sektorale stoffspezifische EU-Umweltrecht (z.B. EU Chemikalien- und Pflanzenschutzrecht) integriert und das Ziel eines guten Zustandes der Meeresumwelt bis 2020 verfolgt.

Das EU-Recht ist folglich durch anspruchsvolle Schutzziele für die marine Umwelt gegenüber Schadstoffen gekennzeichnet. Es soll ein guter Zustand der Küstengewässer bis zum Jahr 2015 (EU WRRL) und ein guter Zustand der europäischen Meere bis zum Jahr 2020 (EU-Meeresstrategie-Rahmen-RL) erreicht werden. Die Schutzzielbestimmungen spiegeln teilweise auch die internationalen Konventionen zum Schutz des Nordost-Atlantiks (OSPARCOM) und der Ostsee (HELCOM) wider. Dies gilt insbesondere für die Zielvorgabe der EU Wasserrahmenrichtlinie, Emissionen von gefährlichen Substanzen in die Meeresumwelt stufenweise $\mathrm{zu}$ reduzieren mit dem ultimativen Ziel, bis zum Jahr 2020 für natürliche Substanzen die natürliche Hintergrundkonzentration und für anthropogene, synthetische Substanzen Konzentrationen nahe Null in der marinen Umwelt zu erreichen (Esbjerg Deklaration 1995; OSPARCOM 1998b; Hagner und Peters 2001). Aus diesen Schutzzielbestimmungen wurde die Notwendigkeit der Implementierung einer prospektiven marinen ökologischen Risikobewertung von Chemikalien, Pestiziden und Baggergut im EU-Recht abgeleitet.

\subsection{Marine ökologische Risikobewertung von Substanzen} in ausgewählten europarechtlichen Regelungen

Es wurde im Folgenden geprüft, ob das EU-Chemikalienund Pflanzenschutzrecht sowie die internationalen und nationalen Regelungen zum Baggergut-Management den Schutz der Meeresumwelt durch eine prospektive marine ökologische Risikobewertung adäquat berücksichtigen. Der Schwerpunkt dieser Analyse richtete sich auf die Berücksichtigung mariner ökotoxikologischer Testverfahren zum hazard assessment.

Marine ökologische Risikobewertung im EU Chemikalienrecht Für die Risikobewertung der Emission von Chemikalien und ihrer Wirkung auf die Struktur und Funktion von marinen Ökosystemen wurden nach dem alten Chemikalienrecht für „,neue“ Chemikalien, Altstoffe und Biozide nach dem EU Technischen Leitfaden für Risikobewertung (TGD, EC 2003) zwei Methoden angewendet:

1. Für die Risikobewertung der Emissionen auf lokaler und regionaler Skala wurde eine quantitative Risikobewer- tung mithilfe der PEC/PNEC-Methode vorgenommen. Dabei wurde die vorhergesagte Umweltkonzentration einer Substanz (Predicted Environmental Concentration) ins Verhältnis zu der vorhergesagten Konzentration gesetzt, unterhalb derer keine schädlichen Effekte auf Organismen auftreten (Predicted No Effect Concentration).

2. Für die Risikobewertung der Emission auf kontinentaler/ globaler Skala wurde ein qualitatives Verfahren durchgeführt. Hierfür erfolgte eine Risikobewertung der Substanzen nach ihren intrinsischen Stoffeigenschaften, der PBT-Bewertung, in Kombination mit einer Bewertung der primären Emissionsquellen. Eine Substanz wird als PBT-Substanz identifiziert, wenn sie ein Potenzial zur Persistenz in der Umwelt, zur Bioakkumulation und Toxizität in Biota aufweist. Darüber hinaus werden auch Substanzen mit sehr persistenten (very persistent) und sehr bioakkumulativen Eigenschaften (very bioaccumulative) $(v P v B)$ erfasst. Für PBT und vPvB Substanzen kann keine ,sichere“ Umweltkonzentration mit hinreichender Zuverlässigkeit bestimmt werden. Denn die Effekte persistenter, bioakkumulierender Substanzen sind langfristig nicht vorhersagbar, sowie räumlich und zeitlich von der Emissionsquelle getrennt.

Diese beiden Methoden der marinen ökologischen Risikobewertung werden prinzipiell auch unter dem neuen europäischen Chemikalienrecht REACH (EG 2006) und ihren Technischen Leitfäden (u. a. ECHA 2007, ECHA 2008) beibehalten. Unter REACH unterliegen alte und neue Chemikalien mit einer Produktionsmenge größer als eine Tonne pro Jahr einer Registrierung, Evaluierung und gegebenenfalls einer Zulassungspflicht. Die vorläufige Risikobewertung durch den Hersteller oder Importeur (Chemical Safety Assessment) erfolgt für Substanzen mit einem Produktionsvolumen größer als 10 Tonnen pro Jahr. Eine vollständige Ermittlung bzw. Abschätzung von Risiken (Chemical Risk Assessment) ist nur für Stoffe vorgesehen, die gefährliche Eigenschaften aufweisen. Die Risikocharakterisierung erfolgt ebenfalls durch einen Vergleich von PEC zu PNEC. Die PNECs eines jeweiligen Umweltkompartiments wurden nach dem alten TGD (EC 2003) und werden auch unter REACH durch Extrapolation von Biotests mithilfe von Sicherheitsfaktoren gewonnen (ECHA 2008). Für die Ableitung der PNECs für marine Organismen werden, wenn keine marinen Toxizitätsdaten vorliegen, limnische Toxizitätsdaten herangezogen und von diesen auf die Sensitivität mariner Organismen extrapoliert. Die Unsicherheiten bei der Extrapolation von Süßwasserdaten auf marine Organismen werden in Abschn. 4 analysiert.

Stoffe, die PBT oder vPvB Eigenschaften haben und somit für die marine Umwelt von großer Bedeutung sind, unterliegen im REACH-System einer Zulassung. Mit der PBTBewertung wurden ähnliche Konzepte internationaler Foren 
Tab. 1 Unterschiedliche Kriterien zur Definition gefährlicher Substanzen. SW Meerwasser; $F W$ Süßwasser (Floeter 2007)

\begin{tabular}{|c|c|c|c|}
\hline & EG PBT-Substanzen & EG vPvB-Substanzen & OSPAR „Hazardous Substances“ \\
\hline $\begin{array}{l}\text { Persistenz/Langlebigkeit bzw. } \\
\text { Abbaubarkeit, } \\
\text { Halbwertszeit [Tage] }\end{array}$ & $\begin{array}{l}\mathrm{SW}>60 \\
\text { SW Sediment }>180 \\
\mathrm{FW}>40 \\
\text { FW Sediment }>120\end{array}$ & $\begin{array}{c}\text { Wasser }>60 \\
\text { Sediment }>180\end{array}$ & Wasser $>50$ \\
\hline Bioakkumulation & BCF $1>2000$ & $\mathrm{BCF} 1>5000$ & $\mathrm{BCF} 1>500$ \\
\hline $\begin{array}{l}\text { Toxizität im Gewässer } \\
{[\mathrm{mg} / \mathrm{L}]}\end{array}$ & $\begin{array}{c}\text { Akut }(\text { LC50) }<0,1 \\
\text { Chronisch }(\text { NOEC })<0,01\end{array}$ & Kein Kriterium & $\begin{array}{c}\text { Akut }(\text { LC50) }<1 \\
\text { Chronisch }(\text { NOEC })<0,1\end{array}$ \\
\hline Toxzität für Mensch und Tier & \multicolumn{3}{|c|}{$\begin{array}{l}\text { Krebserregend, erbgutschädigend, fortpflanzungsschädigend oder andere Effekte, die zeitlich verzögert auftre- } \\
\text { ten, unumkehrbar sind und für die eine unschädliche Dosis nicht mit hinreichender Sicherheit bestimmt werden } \\
\text { kann (z.B. hormonell wirkende Stoffe) }\end{array}$} \\
\hline Quelle & TGD, EC 2003 & TGD, EC 2003 & $\begin{array}{c}\text { List of Substances of Possible Concern, } \\
\text { OSPARCOM } 2003\end{array}$ \\
\hline
\end{tabular}

(z.B. die UNEP Stockholm Konvention über persistente organische Schadstoffe (POPs), die OSPAR Hazardous Substances Strategy) berücksichtigt. Ein Vergleich des alten und des derzeit gültigen EU-Chemikalienrechts mit der OSPARKonvention (OSPARCOM 2003) zeigte jedoch, dass für die Identifikation von PBT- und vPvB-Substanzen zwar dieselben Kriterien angewendet werden, das EU-Chemikalienrecht jedoch höhere Grenzwerte festgelegt hat und somit ein größeres Risiko toleriert (Tabelle 1): Es setzt eine höhere Persistenz (delta 10 Tage), ein größeres Bioakkumulationspotenzial (Faktor 4-10) und eine höhere Toxizität (Faktor 10) voraus, bevor es die Substanz als PBT klassifiziert. Darüber hinaus sind unter REACH die Testverfahren, die eine Beurteilung der Substanzen nach den PBT-Kriterien erlauben, erst ab einer Tonnage von insgesamt mehr als 100 t/a gefordert (Steinhäuser und Füll 2004; Schulte und PeltolaThies 2004). Damit wird ein wesentlicher Regelungsfaktor, der für den Schutz der marinen Umwelt entscheidend ist, faktisch ausgehebelt.

Marine ökologische Risikobewertung im EU-Zulassungsverfahren von Pestiziden Derzeit befindet sich das EU-Pestizidrecht in einem Reformprozess (u.a. EC 2006a,b,c). In der derzeit geltenden Richtlinie 91/414/ EWG über das Inverkehrbringen von Pflanzenschutzmitteln (EWG 1991) wird der Schutz der marinen Umwelt nicht ausdrücklich genannt, jedoch werden in dem neuen Vorschlag für eine Revision der EU Richtlinie 91/414/ EWG durch eine EU Verordnung (EC 2006b) Mündungsund Küstengewässer explizit in der Schutzzielbestimmung berücksichtigt.

Entsprechend wird die marine Umwelt auch in der ökologischen Risikobewertung nach der derzeit gültigen RL 91/414 EWG (EWG 1991) nicht gesondert betrachtet. Der für die aquatische Umwelt erforderliche ökotoxikologische Prüfumfang umfasst ausschließlich limnische Biotests (Peters 1999). Eine marine ökologische Risikobewertung, in der die Ökotoxizität der Pestizide gegenüber Meerwasser- organismen bestimmt wird, ist leider auch nicht im Rahmen der Revision der EU RL 91/414 EWG geplant. Die neue EU-Verordnung, die in erster Lesung im EU-Parlament im Oktober 2007 beschlossen wurde, fordert aber eine qualitative marine ökologische Risikobewertung: Wirkstoffe mit PBT- oder vPvB-Eigenschaften dürfen nicht zugelassen werden. Die Kriterien für die Klassifizierung der Wirkstoffe als PBT- oder vPvB-Substanzen stimmen bezüglich der aquatischen Umwelt mit denen des TGD (2003) (siehe Tabelle 1) überein. Vergleicht man dies mit der derzeit gültigen RL 91/414 EWG (EWG 1991), so sind nun erstmals auch Meerwasserbedingungen einschließlich mariner Sedimente berücksichtigt. Darüber hinaus dürfen persistente organische Schadstoffe nicht als Wirkstoffe zugelassen werden. Als Kriterium wird neben der Persistenz und dem Bioakkumulationspotenzial auch das Potenzial zum Transport in der Umwelt über weite Entfernungen herangezogen. Erstmals wird folglich im Rahmen des Zulassungsverfahrens von Pestiziden auch der Schadstoffferntransport berücksichtigt.

Die Revision des EU-Zulassungsverfahrens für Pflanzenschutzmittel ist aus Sicht des Meeresumweltschutzes als Fortschritt zu sehen. Jedoch erfüllen die Grenzwerte für die PBT-Eigenschaften nicht die internationalen Anforderungen durch OSPARCOM (siehe Tabelle 1). Darüber hinaus werden keine ökotoxikologischen Studien mit Meerwasserorganismen gefordert, obwohl gezeigt wurde, dass marine Organismen aufgrund der physiko-chemikalischen Eigenschaften von Pestiziden sensitiver als limnische Organismen reagieren können (Wheeler et al. 2002).

Marine ökologische Risikobewertung von Baggergut in Küstengewässern Bisher gibt es keine europarechtliche Regelung für das Baggergut-Management. Sedimente als Bestandteil der Gewässer sind in begrenztem Umfang Gegenstand der EU-Wasserrahmenrichtlinie und EU-Meeresstrategie-Rahmenrichtlinie. Baggergut-Management ist jedoch aus europarechtlicher Sicht primär Umgang mit Abfall 
(EWG 1975; EC 2000), die Berücksichtigung von Sedimenten als Ressource, so wie es in internationalen Richtlinien zum Baggergut-Management vorgesehen ist, wird im Europarecht weitestgehend vernachlässigt.

Im Rahmen von internationalen Übereinkommen zum Schutz der Meeresumwelt wurden Richtlinien zum Baggergut-Management entwickelt (HELCOM 1992; PIANC 1997; 1998, OSPARCOM 1998; LC 2000). Diese sehen für die Risikoabschätzung eine biologische Charakterisierung mit ökotoxikologischen Methoden vor. Folgende Aspekte sollten, sofern geeignet, bestimmt werden: Akute Toxizität, chronische Toxizität und das Bioakkumulationspotenzial (HELCOM 1992; PIANC 1997 und 1998, OSPARCOM 1998a, LC 2000). Darüber hinaus wird zum Teil auch die Erfassung der Mutagenität und Genotoxizität gefordert (PIANC 1998). Die ökotoxikologischen Methoden werden von OSPARCOM weiter konkretisiert: Ein Biotest-Set bestehend aus 2-4 Biotests mit Vertretern unterschiedlicher trophischer Ebenen wird empfohlen. Darüber hinaus können Biomarker, Mikro- und Mesokosmos Experimente sowie Felduntersuchungen der benthischen Lebensgemeinschaft geeignete Methoden zur Effektabschätzung darstellen (OSPARCOM 1998a).

Die nationale Umsetzung der internationalen BaggergutManagement-Richtlinien für die Wasser- und Schifffahrtsverwaltung des Bundes (WSV) ist die „Handlungsanweisung für den Umgang mit Baggergut im Küstenbereich“ (HABAK-WSV, BfG 1999). Die ökotoxikologischen Untersuchungen umfassen laut derzeit gültiger HABAK-WSV nur die Durchführung des Leuchtbakterientests (nach DIN 38412 Teil 34). Aufgrund der im Rahmen dieser Arbeit durchgeführten Standardisierung und Validierung mariner Biotests (Abschn. 5), werden in Ergänzung zum Leuchtbakterientest seit ca. 2005 routinemäßig auch der akute Amphipodentest (DIN EN ISO 16712) und der marine Algentest (DIN EN ISO 1053) zur ökotoxikologischen Charakterisierung von Meerwasser- und Brackwassersedimenten eingesetzt.

\section{Besonderheiten der Schadstoffexposition und Wirkung in der marinen Umwelt im Vergleich zu limnischen Ökosystemen}

Auch aus naturwissenschaftlicher Sicht ist aufgrund der Besonderheiten der Schadstoffexposition und Wirkung in der marinen Umwelt im Vergleich zu limnischen Ökosystemen eine gesonderte marine ökologische Risikobewertung für die Zulassung von Chemikalien und Pestiziden im europäischen Umweltrecht und für das Baggergut-Management notwendig. Hier sollen kurz nur einige Besonderheiten insbesondere bezüglich der Schadstoffwirkung in der marinen Umwelt zusammengefasst werden.
Die Salinität hat weitreichende Auswirkungen auf die Schadstoffexposition und Wirkung in marinen Organismen: Die Salinität verändert die Bioverfügbarkeit von Schwermetallen und unpolaren organischen Substanzen. So wird die Speziation von Metallen durch den pH-Wert, den Härtegrad und die Ionenstärke beeinflusst (Wheeler et al. 2001). Die Löslichkeit unpolarer organischer Schadstoffe wird aufgrund der Aussalzungseffekte um 10-50\% herabgesetzt (ECETOC 2001). Dies ist für die Schadstoffanreicherung in marinen Sedimenten und für die benthische Lebensgemeinschaft von großer Bedeutung: Nicht nur die Ausfällung sondern auch die Zunahme des OctanolWasser-Verteilungskoeffizients $\mathrm{K}_{\mathrm{ow}}$, vermutlich um einen Faktor von 2 (ECETOC 2001), führt zu einer Zunahme der Hydrophobizität und steigert somit das Potenzial der Schadstoffe, sich im Sediment oder in der Biota anzureichern. Die Bedeutung der Exposition benthischer Organismen gegenüber unpolaren organischen Schadstoffen über Sedimentpartikel, durch direkten Körperkontakt mit dem Sediment oder über die benthische Nahrung nimmt somit unter Meerwasserbedingungen im Vergleich zu limnischen Bedingungen zu.

Der abiotische und biotische Abbau ist unter Meerwasserbedingungen aufgrund von u. a. niedrigeren Temperaturen, verringerter Lichtintensität durch hohe Turbulenz und hohe Resuspension von Sedimentpartikeln (ECETOC 2001) sowie geringerer Bakteriendichte (BUA 2000) herabgesetzt. Die genannten Faktoren führen zu einer stärkeren Anreicherung von unpolaren organischen Schadstoffen in marinen Sedimenten.

Salzgehaltsschwankungen können $\mathrm{zu}$ osmotischem Stress führen, der u.U. zu einer Erhöhung der Sensitivität bestimmter Arten gegenüber Schadstoffen führen kann (u. a. Hall und Anderson 1995; Breitholtz et al. 2001). Auch haben marine Säugetiere im Vergleich zu landlebenden Warmblütern eine erhöhte Stoffwechselaktivität (Tardent 1993), die u. U. durch den Schadstoff-Stoffwechsel zusätzlich gesteigert werden kann (Verslycke 2004). Der Einfluss einer erhöhten Stoffwechselaktivität auf die Sensitivität der marinen Organismen gegenüber Schadstoffen ist noch ungeklärt.

Die durchschnittlich niedrigeren Temperaturen im Meerwasser implizieren verschiedene Anpassungsmodi, die zu unterschiedlicher Schadstoffwirkung in Meerwasserorganismen im Vergleich zu limnischen Organismen führen kann: Großwüchsigkeit der Organismen, lange Lebenszeiten (K-Strategen) der Top-Prädatoren im Nahrungsnetz sowie Fettdepots können zur erhöhten Schadstoffanreicherung durch Bioakkumulation und Biomagnifikation führen. Die in den Fettdepots angereicherten lipophilen Schadstoffe, z. B. in Eisbären, können in Zeiten der Futterarmut oder der Stillzeit von Jungtieren mobilisiert und auf die Jungtiere übertragen werden (O’Hara und Becker 2003). 


\section{Kann die Sensitivität von marinen Organismen gegenüber Narkotika durch die Sensitivität von Süßwasser-Organismen vorhergesagt werden?}

Wird das europäische Chemikalienrecht den Besonderheiten der Schadstoffwirkung unter Meerwasserbedingungen aus naturwissenschaftlicher Sicht gerecht? Nach den technischen Leitfäden des alten und neuen EU-Chemikalienrechts (TGD, EC 2003; ECHA 2008) werden aufgrund der geringen Anzahl von Meerwasser-Ökotoxizitätsdaten Süßwasserökotoxizitätsdaten als Substituenten zur Ableitung der PNEC für marine Organismen herangezogen. Wenn keine marinen Toxizitätsdaten vorliegen, wird von dem sensitivsten Süßwassertoxizitätstest unter Berücksichtigung eines Sicherheitsfaktors von 10 auf die Sensitivität von marinen Organismen extrapoliert. Mit welchen Unsicherheiten ist diese Extrapolation behaftet? Dies wurde exemplarisch für die Stoffklasse der Narkotika im Rahmen eines CEFIC LRI Vorhabens geprüft. Narkotika bewirken eine unspezifische, reversible Störung der Zellmembranfunktion, die auch zum Tode der Organismen führen kann (van Wezel und Opperhuizen 1995). $60 \%$ der Industriechemikalien die in die aquatische Umwelt gelangen gehören zur Substanzklasse der Narkotika (van Wezel und Opperhuizen 1995). Es wurden unpolare Narkotika (Mode of Action 1) und polare Narkotika (Mode of Action 2) (Verhaar et al. 1992) untersucht. Aufgrund der unspezifischen Wirkweise der Narkotika wurde die Hypothese aufgestellt und geprüft, dass es keinen signifikanten Unterschied zwischen der Sensitivität von Süßwasserorganismen und Meerwasserorganismen gegenüber Narkotika gibt.

Es wurden für 64 Narkotika Süßwasser- (FW) und Meerwasser- (SW) Toxizitätsdaten aus verschiedenen Datenbanken zu einer „MarSens“ Datenbank zusammengefasst (Peters et al. 2005; Floeter et al. 2008). Insgesamt enthielt die „MarSens“ Datenbank 2652 marine Toxizitätsdaten. Obwohl in der Datenbank insgesamt 14 marine taxonomische Gruppen vertreten waren, dominierten die Daten von Fischen und Crustacea (zusammen 75\%). Folglich erlaubte die Verteilung der Daten nur einen direkten Vergleich zwischen den taxonomischen Gruppen: Fische, Krebse und Algen. Es wurden hauptsächlich akute Toxizitätsdaten mit unspezifischen Endpunkten (Mortalität) genutzt. Nur 4\% der marinen Toxizitätsdaten waren chronische Daten, hauptsächlich basierten diese auf den marinen Algenwachstumshemmtests. Darüber hinaus war die Qualität der ToxizitätsDaten dadurch begrenzt, dass sie nicht für den Vergleich der SW- und FW-Sensitivität hergestellt wurden. Somit variierten sie neben den gewollten Unterschieden, die mit den verschiedenen Lebensräumen verbunden sind (z. B. Art, Salinität und pH-Wert), auch in anderen Parametern, wie z. B. in Dauer der Exposition.

Zur Prüfung der Frage, ob die Sensitivitäten von Süßwasserorganismen gegenüber Narkotika als Stellvertreter für
Meerwasserorganismen fungieren können, wurde die lineare Regressionsanalyse (Model II) angewandt (Peters et al. 2005, Floeter et al. 2008). Ziel war es, eine Aussage für die Substanzklasse (getrennt nach MoA 1 und MoA 2) auf Basis der selektierten Narkotika machen zu können. Deshalb wurden für jede Substanz die geometrischen Mittelwerte aus den Toxizitätsdaten, für Süßwasser- und Meerwassertoxizitätsdaten getrennt, gebildet und eine lineare Regressionsanalyse für die Gesamtheit der ausgewählten Substanzen eines MoA durchgeführt (Peters et al. 2005; Floeter et al. 2008). Es wurde auch das untere einseitige 95\%-Vorhersage-Intervall der Regressionsgeraden bestimmt. Dieses Maß ist zugehörig zu der zugrundeliegenden Sensitivitätsverteilung und teilt von dem gesamten Bereich der Meerwasserdaten eine $5 \%$ Region unterhalb der Regressionsgeraden ab, unter der die sensitivsten Meerwasserarten gefunden werden. Es ist somit äquivalent zu dem HC5-Bereich (Hazardous Concentration für 5\% der Arten) (Peters et al. 2005, Floeter et al. 2008).

Die lineare Regression für alle MoA-1-Substanzen ( $\mathrm{n}=$ 46) der SW- und FW-Organismen unter der gemeinsamen Berücksichtigung aller taxonomischen Gruppen zeigte, dass die SW- und FW-Sensitivitäten signifikant korrelierten (Peters et al. 2005, Floeter et al. 2008). Dieses Ergebnis wurde auch für alle MoA-2-Substanzen unter der gemeinsamen Berücksichtigung aller taxonomischen Gruppen gewonnen. Folglich ist, auf der Basis der zugrundeliegenden Datenbank und unter der vereinten Berücksichtigung aller taxonomischen Gruppen, die Extrapolation der SW-Sensitivität von der FW-Sensitivität gegenüber Narkotika gerechtfertigt. Darüber hinaus würde in den Fällen, in denen die taxonomischen Gruppen vereint berücksichtigt wurden, ein Extrapolationsfaktor von 10 ausreichen um $95 \%$ der marinen Organismen gegenüber MoA-1- und MoA-2-Substanzen zu schützen (Peters et al. 2005, Floeter et al. 2008).

Auch die Regressionsanalysen für MoA-1-Substanzen getrennt nach den taxonomischen Gruppen zeigten für Fische und Crustacea, dass es keinen signifikanten Unterschied zwischen der FW- und SW-Sensitivität gab (Peters et al. 2005, Floeter et al. 2008). Die Regressionsgerade der SW-Algen gegen die FW-Algen hingegen hatte eine Steigung $(\mathrm{b} 1=0,388)$ signifikant größer als null $(\mathrm{p}=0,047)$ aber auch signifikant kleiner als eins $(p=0,00045)$. Dass die Steigung der Regressionsgeraden signifikant kleiner als eins ist, kann als Hinweis gedeutet werden, dass die marinen Algen eine signifikant höhere Sensitivität gegenüber den untersuchten MoA-1-Substanzen $(n=20)$ als die Süßwasseralgen haben. Neben biologischen Unterschieden zwischen Süßwassergrünalgen und Meerwasser-Diatomeen wurde auch eine unterschiedliche Bioverfügbarkeit der unpolaren Narkotika unter Meerwasserbedingungen als Erklärungsansatz herangezogen. Aufgrund des geringen Bestimmtheitsmaßes $\left(r^{2}=0,20\right)$ der Regression sollte dieses Ergebnis je- 
doch durch weitere Experimente geprüft werden (Peters et al. 2005, Floeter et al. 2008).

Die Regressionsanalysen, welche getrennt nach den taxonomischen Gruppen durchgeführt wurden, zeigten für alle MoA-1- und MoA-2-Substanzen eine mittlere, delogarithmierte Distanz (MD) zwischen der Regressionsgeraden und dem HC5-Bereich in der Spanne von MD = 5,63 bis $\mathrm{MD}=91,69$, wobei der höchsten Wert für Algen ermittelt wurde. Für die Extrapolation der Sensitivität von Süßwasseralgen auf Meerwasseralgen auf Basis der Regressionsgeraden würde demnach ein Sicherheitsfaktor von 92 notwendig sein, um $95 \%$ der untersuchten Meerwasseralgen zu erfassen (Peters et al. 2005; Floeter et al. 2008).

Da Hinweise auf signifikante Unterschiede in der relativen Toxizität von Narkotika gegenüber FW-und SW-Taxa festgestellt wurden, ist die Berücksichtigung der marinen Biodiversität in der Datenbasis von herausragender Bedeutung. Die marine Umwelt umfasst ca. 78\% der aquatischen Arten (Peters et al. 2005) und 15 exklusiv marine Stämme (TGD, EC 2003). Die öffentlich zugänglichen Toxizitätsdatenbanken spiegeln die marine Biodiversität nicht wider. Daten von bedeutenden marinen Taxa, z. B. Mollusca, und exklusiv marinen Taxa, z. B. Echinodermata oder Tunicata, fehlen.

Es wurden für die Bewertung der Sensitivität von Süßwasser- und Meerwasserorganismen gegenüber Narkotika aus Ermangelung an Sedimenttestergebnissen nur Daten der Wasserphasetests herangezogen. Wie bei den Besonderheiten der Schadstoffwirkung im marinen Bereich ausgeführt, reichern sich unpolare Narkotika aufgrund der Aussalzungseffekte, der Zunahme der Hydrophobizität und des geringeren Abbaus insbesondere in marinen Sedimenten an. Für Narkotika mit mit einem $\log \mathrm{k}_{\mathrm{ow}}>4,5$ sollten deshalb andere Expositionspfade, über die Nahrung und über Sedimentpartikel, mit marinen benthischen Organismen un- tersucht werden. Es fehlte zu Beginn dieser Arbeit jedoch an international standardisierten marinen Sedimentbiotests, insbesondere chronischen Gesamtsedimenttests (OSPARCOM 1998a; TGD, EC 2003). Neben der Stoffbewertung von Chemikalien und Pestiziden ist der Einsatz mariner Sedimentbiotests auch für die Bewertung der Qualität von Meer- und Brackwasser-Sedimenten erforderlich.

\section{Weiterentwicklung und Standardisierung eines minimalen biologischen Testsets zur Bewertung von aestuarinen und marinen Sedimenten}

Im Rahmen eines Vorhabens des Umweltbundesamtes (UBA) (im Zeitraum von 1999-2002) wurde erstmals ein marines Biotest-Set zur Bewertung von Meer- und Brackwasser-Sedimenten innerhalb Deutschlands validiert, harmonisiert und in nationalen Laboratorien implementiert. Für die angestrebte Implementierung des Testsets im nationalen und europäischen Umweltrecht war die nationale und internationale Standardisierung der Testverfahren von groBer Bedeutung (Peters et al. 2002; Peters und Ahlf 2003).

Das Testset (Abb. 2) besteht aus zwei Tests für die Wasserphase (Eluate): dem Leuchtbakterientest und dem marinen Algentest mit Phaeodactylum tricornutum sowie dem Gesamtsedimenttest mit dem Amphipoden Corophium volutator. Damit wurden zwei akute Tests und ein chronischer Test ausgewählt, die als internationale (ISO) und nationale (DIN) Standards für andere Anwendungsbereiche vorlagen (DIN EN ISO 11348-1-3, 1999; DIN EN ISO 10253, 1998, 2006) bzw. sich am Anfang des ISO-Standardisierungsverfahrens (akuter Amphipodentest (ISO 16712, 2007)) befanden. Diese mussten jedoch für ihre Anwendung auf Brack- und Meerwassersedimente weiterentwickelt werden (Peters und Ahlf 2003). Dafür war eine Modifikation
Abb. 2 Das minimale marine Biotest-Set (Floeter 2007)

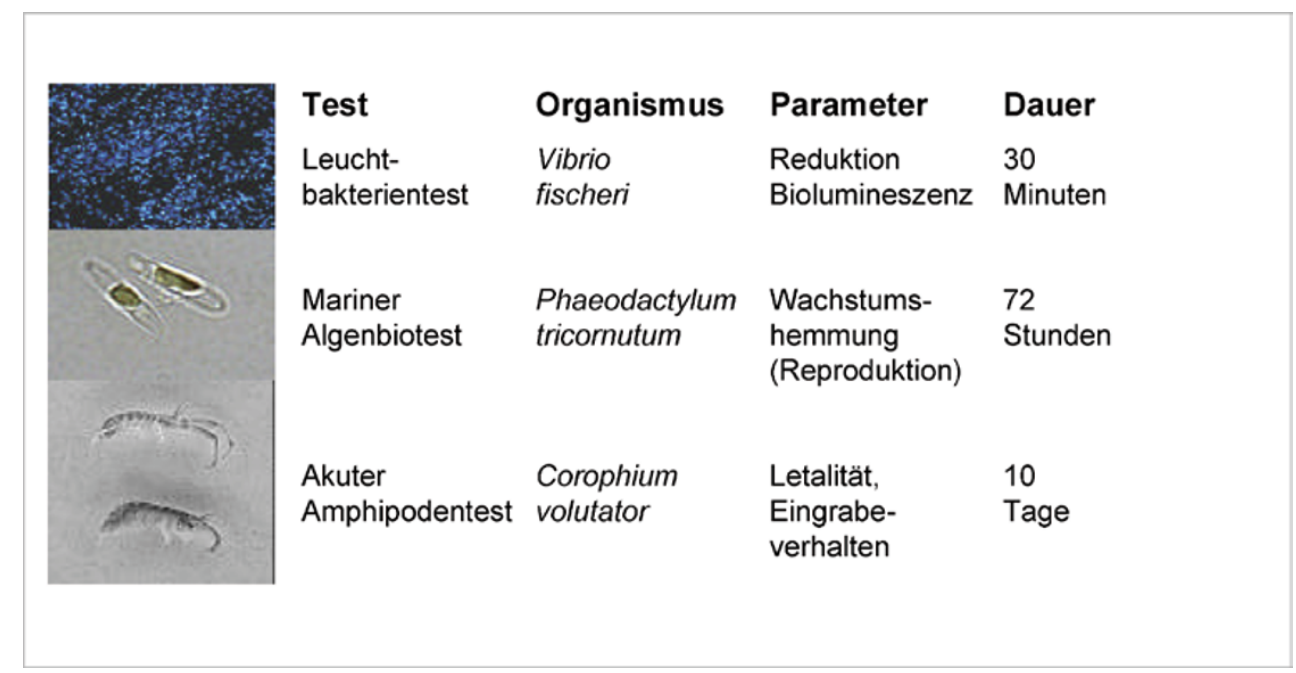


des Leuchtbakterientests notwendig, da bei Anwendung der Testvorschrift unter Brack-/Meerwasserbedingungen Fördereffekte auftraten, die Hemmungen maskieren können. Die Modifikation des Leuchtbakterientests wurde in einem deutschlandweiten Ringtest unter Beteiligung von 24 Laboratorien validiert. Darüber hinaus wurde die Testvorschrift des marinen Algentests von der Einzelsubstanzprüfung auf die Testung von Meer- und Brackwasser-Eluate erweitert. Mit dieser Arbeit wurde auch der akute Amphipodentest im ISO-CEN-DIN-Standardisierungsverfahren (DIN EN ISO 16712, 2007) maßgeblich unterstützt (Peters und Ahlf 2003).

Das Testset wurde im Rahmen dieser Arbeit erfolgreich in vier Laboratorien etabliert: der Technischen Universität Hamburg-Harburg (TUHH), den Dr. Noack-Laboratorien für Angewandte Biologie, der Bundesanstalt für Gewässerkunde (BfG) und dem (ehemaligen) Niedersächsischen Landesamt für Ökologie (NLÖ). Die Probenahme, Probenvorbereitung, Eluatherstellung und Testdurchführung wurde sowohl zwischen den Testverfahren als auch zwischen den Laboratorien harmonisiert. Die Harmonisierung der Verfahren zwischen den Laboratorien und die Validität des Testsets wurden durch zwei Ringtests an Ostsee- und NordseeSedimentproben nachgewiesen. Darüber hinaus wurde der akute Amphipodentest auch auf EU-Ebene im Rahmen des EU-BEQUALM-Projekts validiert. In der Zwischenzeit haben noch weitere Laboratorien in Deutschland das Testset aufgebaut.
Die Ergebnisse dieser Arbeit sind direkt in die DIN-, CEN- und ISO-Standardisierung gemündet (Peters und Ahlf 2003): Die Weiterentwicklungen der Testverfahren für die Anwendung auf Brack- und Meerwasser-Eluate wurden bei der Revision in die ISO-EN-DIN-Standards $(2006,2008)$ implementiert. Damit steht ein national und international standardisiertes marines Biotest-Set auch für das europäische Chemikalien- und Pflanzenschutzrecht bereit. Das Testset wird seit ca. 2005 routinemäßig für das BaggergutManagement im Rahmen der HABAK-WSV (BfG 1999) angewandt.

\section{Entwicklungen für einen ästuaren und marinen chronischen Gesamtsedimenttest mit Corophium volutator}

Der akute Amphipodentest basierte bis dato auf Wildfängen von Corophium volutator. Da dies aber mit einer unterschiedlichen Vorbelastung der Freilandtiere, der nicht gewährleisteten Verfügbarkeit im Winter sowie mit einem Eingriff ins Ökosystem verbunden war, wurde der Schlickkrebs Corophium volutator im Rahmen dieser Arbeit erstmals über einen Zeitraum von einem Jahr zur Reproduktion unter Laborbedingungen gebracht (Peters und Ahlf 2005). Es gelang, die Tiere nicht nur im Sommer und Herbst sondern auch, entgegen ihrem natürlichen Lebenszyklus, im Winter zur Reproduktion zu bringen. Die Anzahl der Nach-

\begin{abstract}
Abb. 3 Corophium volutator Reproduktionsexperimente: Anzahl Nachkommen pro Weibchen in Abhängigkeit von der Temperatur (Versuchsaufbau 1A, Sommerexperiment, $\mathrm{N}=30$ Weibchen je Temperatur) (Peters und Ahlf 2005)
\end{abstract}

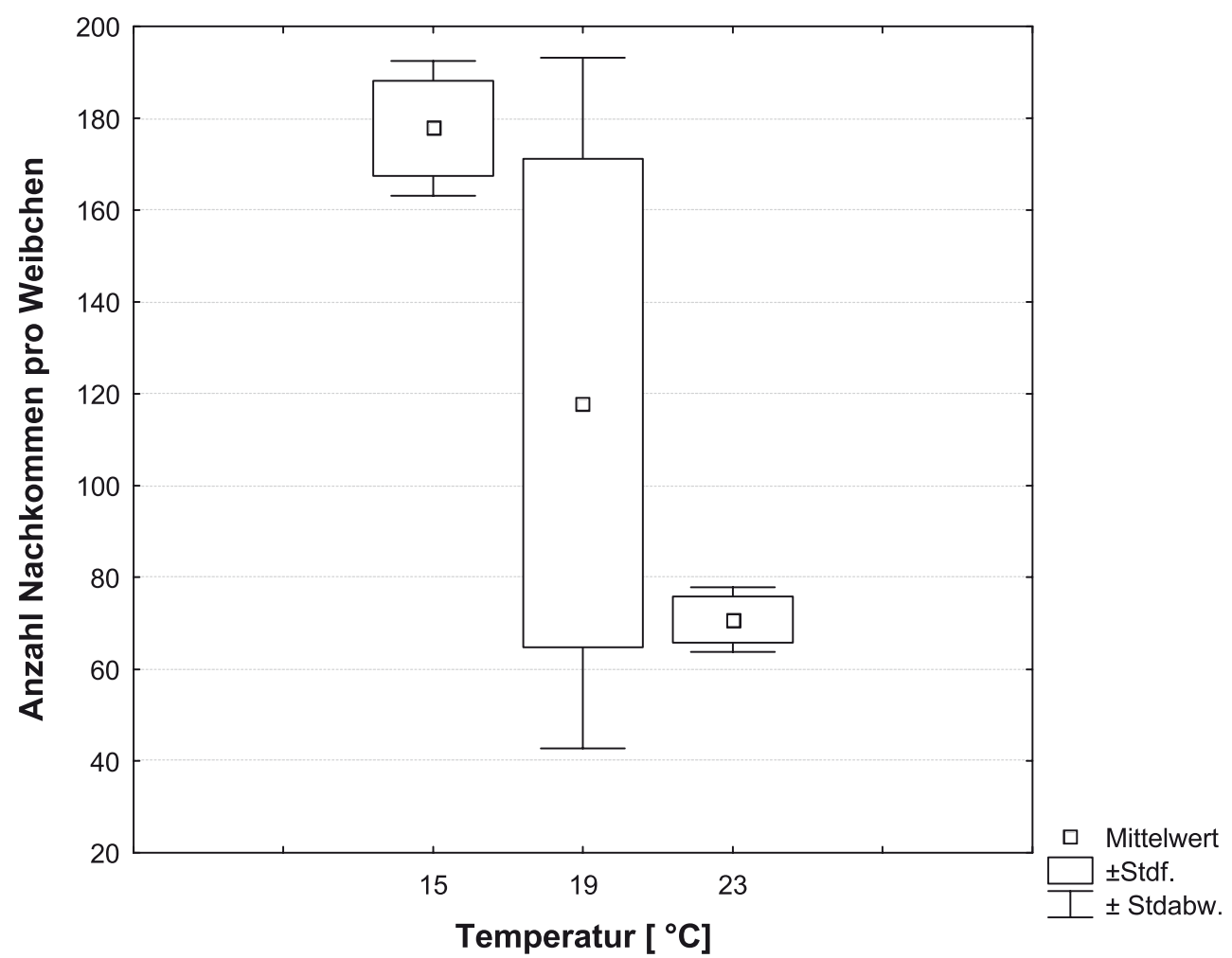


kommen pro Weibchen war bei konstanten Temperatur- und Lichtverhältnissen im Sommer, Herbst und Winter nicht signifikant verschieden. Temperaturversuche zeigten, dass bei $15^{\circ} \mathrm{C}$ mit einer mittleren Anzahl von 178 Nachkommen pro Weibchen ein signifikant größerer Bruterfolg als bei $23^{\circ} \mathrm{C}$ (71 Nachkommen pro Weibchen) $(\mathrm{N}=30$ Weibchen je Temperatur; $t$-test, $p=0,01$ ) erzielt werden konnte (Abb. 3). Die im Labor ermittelte mittlere Wachstumsrate $\left(0,07 \mathrm{~mm} /\right.$ Tag bei $\left.15^{\circ} \mathrm{C}\right)$ entspricht den im Freiland ermittelten Wachstumsraten $(0,05-0,08 \mathrm{~mm} / \mathrm{Tag}$; Möller und Rosenberg 1982; Peer et al. 1986). Die Fitness der gezüchteten Tiere wurde durch langfristige Hälterungen, durch Toxizitätstests und morphologische Prüfungen auf Parasitenbefall überprüft. Ein Vergleich der Sensitivität von Freiland- und Zucht-Tieren gegenüber Ammonium zeigte, dass die ZuchtTiere etwas weniger sensitiv als die Freilandtiere waren, die Sensitivität jedoch im Schwankungsbereich der Freiland-Tiere lag $\left(\mathrm{EC}_{50}\right.$-Wert Zuchttiere $63,92 \mathrm{mg} / 1(95 \% \mathrm{CI}$ $30,34-126,7 \mathrm{mg} / 1, \mathrm{~N}=12$ Tests); $\mathrm{EC}_{50}$-Wert Freilandtiere $40,29 \mathrm{mg} / 1$ (95\% CI 23,66-68,63 mg/l, N = 3 Tests)).

Mit der erfolgreichen Reproduktion und Kultivierung des Schlickkrebses im Labor wurde ein unter standardisierten Bedingungen gezüchteter Testorganismus, der das ganze Jahr verfügbar ist, gewonnen. Sie stellt somit einen wichtigen Beitrag für die Implementierung des Tests dar. Der Einsatz gezüchteter Corophien als Testorganismen wurde daraufhin auch in dem internationalen ISO-Standard berücksichtigt. Darüber hinaus wurde mit der Reproduktion von Corophium im Labor die essentielle Basis für die Entwicklung eines chronischen Gesamtsedimenttests mit den Parametern Anzahl der Nachkommen und Längenwachstum gelegt. Damit wurde auch internationalen Forderungen nach einem chronischen Gesamtsedimenttest (OSPARCOM 1998a; TGD, EC 2003) nachgekommen.

\section{Empfehlungen für die Implementierung der marinen ökologischen Risikobewertung in das europäische Umweltrecht}

Die juristische Analyse kann zusammengefasst werden, dass das EU-Recht zum Meeresumweltschutz durch anspruchsvolle Schutzziele für die marine Umwelt gegenüber Schadstoffen gekennzeichnet ist. Deren Konkretisierung und Umsetzung ist jedoch vom Engagement der Mitgliedstaaten abhängig. Für die Erreichung eines guten Zustandes der Meeresumwelt bis zum Jahr 2020 ist ein qualitativ hochwertiges, über Sektoren und Mitgliedstaaten integrierendes Konzept erforderlich. Dies sollte auch das Baggergut-Management innerhalb der EU Meeresgebiete berücksichtigen. Das sektorale europäische Umweltrecht, das EU-Chemikalien- und das EU-Pflanzenschutzrecht kommen bisher diesen und auch völkerrechtlichen Ansprüchen nur unzureichend nach. Im Rahmen der Implementierung von REACH und der Revision des EU-Pflanzenschutzrechts sollten die PBTKriterien den völkerrechtlichen Vorgaben durch OSPARCOM angepasst werden. Darüber hinaus sollten im Rahmen der Implementierung von REACH die PBT-Testanforderungen für Chemikalien mit einem Volumen weniger als $100 \mathrm{t} / \mathrm{a}$ erhöht werden. Der Wissenszuwachs über die Wirkung von Chemikalien und Pflanzenschutzmitteln in marinen/aestuarinen Organismen unter Brack- und Meerwasserbedingungen sollte gefördert und auch rechtlich gefordert werden, um die Unsicherheit in der Risikobewertung durch Extrapolation von Süßwassertoxizitätsdaten weiter reduzieren zu können. Marine ökotoxikologische Testverfahren, insbesondere marine chronische Gesamtsedimenttests und Testverfahren mit exklusiv marinen Stämmen, sollten in der ökologischen Risikobewertung des EU-Pflanzenschutzrechts und des EUChemikalienrechts verstärkt integriert werden.

Danksagung Besonderer Dank gilt: Prof. Dr. Hans-Joachim Koch, Prof. Dr. Ulrich Förstner, PD Dr. Rüdiger Berghahn, DIN AK 5.3 Marine Biotests, Waldemar Bülow, Steffi Pfitzner, Dr. Udo Noack, DIN AK 5.1 Biotests, Dr. Kristina Barz, Silke Hardtke, Kristin Rosenkranz, Helga von Lochow, PhD Ali Temara, Martin Holt und dem Umweltbundesamt sowie CEFIC LRI für die Förderung.

\section{Literatur}

Beratergremium für Altstoffe (BUA) (2000) Risk assessment für den marinen Bereich (Vorschlag des BUA). In: Behret H (Hrsg) Hirzel Wissenschaftliche Reihe, Nr. 220.S. Hirzel Verlag, Stuttgart Leipzig

Breitholtz M, Hill C, Bengtsson BE (2001) Toxic substances and reproductive disorders in Baltic fish and crustaceans. Ambio 30(45):210-216

Bundesanstalt für Gewässerkunde (BfG) (1999) Handlungsanweisung für den Umgang mit Baggergut im Küstenbereich (HABAKWSV). Bundesanstalt für Gewässerkunde, Koblenz

DIN EN ISO 16712 (2007) Wasserbeschaffenheit - Bestimmung der akuten Toxizität mariner Sedimente oder von Sedimenten aus Flussmündungsgebieten gegenüber Amphipoden (ISO 16712:2005). Beuth Verlag GmbH, Berlin

DIN EN ISO 10253 (1998 und 2006) Wachstumshemmtest mit marinen Algen Skeletonema costatum und Phaeodactylum tricornutum. Beuth Verlag GmbH, Berlin

DIN EN ISO 11348-1-3, (1999 (2008 in Vorbereitung)) Bestimmung der Hemmwirkung von Wasserproben auf die Lichtemission von Vibrio fischeri (Leuchtbakterientest). Normenausschuß Wasserwesen (NAW) im DIN. Beuth Verlag GmbH, Berlin

ECETOC (2001) Risk assessment in marine environments. ECETOC, Technical Report, 82, Brussels

Ebinghaus R, Temme C (2006) Ausbreitung von Schadstoffen in die Polarregionen. In: Lozán JL, Graß1 H, Hubberten H-W, Hupfer P, Karbe L, Piepenburg D (Hrsg) Warnsignale aus den Polarregionen, Wissenschaftliche Auswertungen, Hamburg

European Chemicals Agency (ECHA) (2007) Guidance for the implementation of REACH - guidance for the preparation of an annex $\mathrm{XV}$ dossier on the identification of substances of very high concern, June 2007 (http://reach.jrc.it/guidance_en.htm)

European Chemicals Agency (ECHA) (2008) Guidance for the implementation of REACH - guidance on information requirements and chemical safety assessment chapter R.10: characterisation of 
dose [concentration]-response for environment. May 2008 (http:// reach.jrc.it/guidance_en.htm)

Europäische Kommission (EC) (2002) Mitteilung der Kommission an den Rat, das Europäische Parlament und den Wirtschafts- und Sozialausschuss: Hin zu einer thematischen Strategie zur nachhaltigen Nutzung von Pestiziden. Kom (2002) 349 endgültig, Brüssel 1.7.2002

Europäische Kommission (EC) (2003) Technical Guidance Document on Risk Assessment in support of Commission Directive 93/67/ EEC on Risk Assessmentfor new notified substances Commission Regulation (EC) No 1488/94 on Risk Assessment for existing substances Directive 98/8/EC of the European Parliament and of the Council concerning the placing of biocidal products on the market. European Commission Joint Research Center, Italy

Europäische Kommission (EC) (2005) Mitteilung der Kommission an den Rat und das europäische Parlament. Thematische Strategie für den Schutz und die Erhaltung der Meeresumwelt. KOM(2005) 504 endgültig, 24.10.2005

Europäische Kommission (EC) (2006a) Mitteilung der Kommission an den Rat, das Europäische Parlament, den Europäischen Wirtschafts- und Sozialausschuss und den Ausschuss der Regionen. Hin zu einer thematischen Strategie zur nachhaltigen Nutzung von Pestiziden. $\mathrm{KOM}(2006) 372$ endgültig, 12.7.2006

Europäische Kommission (EC) (2006b) Vorschlag für eine Verordnung des Europäischen Parlaments und des Rates über das Inverkehrbringen von Pflanzenschutzmitteln (von der Kommission vorgelegt). $\mathrm{KOM}(2006) 388$ endgültig, 12.7.2006

Europäische Kommission (EC) (2006c) Vorschlag für eine Richtlinie des Europäischen Parlaments und des Rates über einen Aktionsrahmen der Gemeinschaft für den nachhaltigen Einsatz von Pestiziden (von der Kommission vorgelegt). $\operatorname{KOM(2006)} 373$ endgültig, 12.7.2006

Europäische Kommission (EC) (2007) Mitteilung der Kommission an das Europäische Parlament, den Rat, den Europäischen Wirtschafts- und Sozialausschuss und den Ausschuss der Regionen „Integrierte Meerespolitik für die Europäische Union“, Blaubuch. KOM (2007) 575 endgültig

Europäische Gemeinschaft (EG) (2000) Richtlinie 2000/60/EG des Europäischen Parlaments und des Rates vom 23. Oktober 2000 zur Schaffung eines Ordnungsrahmens für Maßnahmen der Gemeinschaftspolitik im Bereich der Wasserpolitik. Amtsblatt der Europäischen Gemeinschaft, Nr. L 327:1-72

Europäische Gemeinschaft (EG) (2000) 2000/532/EG: Entscheidung der Kommission vom 3. Mai 2000 zur Ersetzung der Entscheidung 94/3/EG über ein Abfallverzeichnis gemäß Artikel 1 Buchstabe a) der Richtlinie 75/442/EWG des Rates über Abfälle und der Entscheidung 94/904/EG des Rates über ein Verzeichnis gefährlicher Abfälle im Sinne von Artikel 1 Absatz 4 der Richtlinie 91/689/ EWG über gefährliche Abfälle (Text von Bedeutung für den EWR). Amtsblatt der Europäischen Gemeinschaft L 226:3-24

Europäische Gemeinschaft (EG) (2006) Verordnung (EG) Nr. 1907/2006 des Europäischen Parlaments und des Rates vom 18. Dezember 2006 zur Registrierung, Bewertung, Zulassung und Beschränkung chemischer Stoffe (REACH), zur Schaffung einer Europäischen Agentur für chemische Stoffe, zur Änderung der Richtlinie 1999/45/EG und zur Aufhebung der Verordnung (EWG) Nr. 793/93 des Rates, der Verordnung (EG) Nr. 1488/94 der Kommission, der Richtlinie 76/769/EWG des Rates sowie der Richtlinien 91/155/EWG, 93/67/EWG, 93/105/EG und 2000/21/ EG der Kommission. Amtsblatt der Europäischen Union, Nr. L $396 / 1$

Europäische Gemeinschaft (EG) (2008) Richtlinie 2008/56/EG des Europäischen Parlaments und des Rates vom 17. Juni 2008 zur Schaffung eines Ordnungsrahmens für Maßnahmen der Gemeinschaft im Bereich der Meeresumwelt (Meeresstrategie-Rahmenrichtlinie). Amtsblatt der Europäischen Union, L 164/19
EWG (1975) Richtlinie 75/442/EWG des Rates vom 15. Juli 1975 über Abfälle. Amtsblatt der Europäischen Gemeinschaft L 194: $39-41$

EWG (1991) Richtlinie 91/414/EWG des Rates vom 15. Juli 1991 über das Inverkehrbringen von Pflanzenschutzmitteln. Amtsblatt der Europäischen Gemeinschaft L 230:1-32

Floeter C, Ahlf W, von Lochow HEC, Temara AR, Holt M, Ratte HT (2008) Assessing the uncertainty in the extrapolation of the sensitivity of freshwater to saltwater organisms exposed to narcotics (submitted)

Floeter C (2007) Entwicklung von ökotoxikologischen Instrumenten und ihre rechtliche Implementierung zur marinen ökologischen Risikobewertung von Chemikalien, Pestiziden und Baggergut. Shaker Verlag, Aachen

Gregory M, Cyr D (2003) Effects of environmental contaminants on the endocrine system of marine mammals. In: Vos JG, Bossart GD, Fournier M, O'Shea TJ (Hrsg) Toxicology of Marine Mammals. (Series: New Perspectives: Toxicology and the environment), vol 1(4). Taylor \& Francis, London New York, S 67-81

Hagner C, Peters C (2001) International regulations concerning dredged material management. In: Gandrass J, Salomons W (Hrsg) Dredged material in the port of Rotterdam - Interface between Rhine Catchment Area and North Sea. Project Report, GKSS Research Centre, Geesthacht, S 214-229

Hall LJ, Anderson RD (1995) The influence of salinity on the toxicity of various classes of chemicals to aquatic biota. Critical Reviews in Toxicology 25:281-346

Helsinki Commission (HELCOM) (1992) Revised guidelines for the disposal of dredged spoils. HELCOM, Helsinki

Internationale Nordseeschutz-Konferenz (1995) Esbjerg Declaration Ministerial Declaration of the Fourth International Conference on the Protection of the North Sea

London Convention (LC) (2000) Specific guidelines for assessment of dredged material. Twenty-second consultative meeting of contracting parties to the convention on the prevention of marine pollution by dumping of wastes and other material 1972. 18-22 September 2000

Moeller RB (2003) Pathology of marine mammals with special reference to infectious diseases. In: Vos JG, Bossart GD, Fournier M, O'Shea TJ (Hrsg) Toxicology of Marine Mammals, Series: New Perspectives: Toxicology and the environment. Taylor \& Francis, London New York, S 3-37

O'Hara TM, Becker PR (2003) Persistent organic contaminants in Arctic marine mammals. In: Vos JG, Bossart GD, Fournier M, O'Shea TJ (Hrsg) Toxicology of Marine Mammals, Series: New Perspectives: Toxicology and the environment, vol 1(8). Taylor \& Francis, London New York, S 206-246

O'Shea TJ, Bossart GD, Fournier M, Vos JG (2003) Perspectives for the future. In: Vos JG, Bossart GD, Fournier M, O'Shea TJ (Hrsg) Toxicology of Marine Mammals, Series: New Perspectives: Toxicology and the environment, vol 1(23). Taylor \& Francis, London New York, S 595-613

Oslo and Paris Commission (OSPARCOM) (1998a) OSPAR guidelines for the management of dredged material. Nr. 1998-20

Oslo and Paris Commission (OSPARCOM) (1998b) Sintra Statement - Ministerial Meeting of the OSPAR Commission on 22.-23. July 1998

Peters C (1999) Die Belastbarkeit von Ökotoxizitätstests aus naturwissenschaftlicher und umweltrechtlicher Sicht - aufgezeigt an Beispielen des Gewässerschutzes. Diplomarbeit, Universität Hamburg

Peters C, Becker S, Noack U, Pfitzner S, Bülow W, Barz K, Ahlf W, Berghahn R (2002) A marine bioassay test set to assess marine water and sediment quality - its need, the approach and first results. Ecotoxicology 11(5):379-384 
Peters C, Ahlf W (2003) Validieren, Harmonisieren und Implementieren eines minimalen biologischen Testsets zur Bewertung mariner Wasser- und Sedimentproben. Umweltbundesamt, Forschungsbericht 29925 261, Berlin

Peters C, Ahlf W (2005) Reproduction of the estuarine and marine amphipod Corophium volutator (Pallas) in laboratory for toxicity testing. Chemosphere 59:525-536

Peters C, Ahlf W, von Lochow HEC, Ratte H-T (2005) How should we deal with the uncertainty in the extrapolation of the sensitivity of marine organisms to narcotics? - Critical review of the state of the art of marine risk assessment and recommendations for future research. MarSens CEFIC LRI-ECO1B-TUHH-0407Final Report

PIANC (1997) Dredged material management guide. Special Report of the Permanent Environmental Commission. In: Vellinga $T$ (Hrsg) Brüssel

PIANC (1998) Management of aquatic disposal of dredged material. Report of Working Group I of the Permanent Environmental Commission. Brüssel

Sachverständigenrat für Umweltfragen (SRU) (2004) Meeresumweltschutz für Nord- und Ostsee - Sondergutachten. Berlin

Sachverständigenrat für Umweltfragen (SRU) (2006) Der Vorschlag der Europäischen Kommission für eine Meeresschutzstrategie Rückzug aus der europäischen Verantwortung? Kommentar zur Umweltpolitik, Nr. 5, April 2006

Schulte C, Peltola-Thies J (2004) Mehr Vorsorge in der Umweltbewertung - Persistenz, Bioakkumulation und Toxizität. Präsentation, Jahrestagung SETAC German Language Branch, Aachen
Steinhäuser KG, Füll C (2004) Hazardous substances and the REACH proposal. Presentation: Towards a European Policy on the Marine Environment. SRU-EEAC 15 Okt. 2004, Berlin

Tanabe S (2004) PBDEs, an emerging group of persistent pollutants. Marine Pollution Bulletin 49:369-370

Tanabe S (2005) Synthetic musks - arising new environmental menace? Marine Pollution Bulletin 50:1025-1026

Tardent P (1993) Meeresbiologie, 2., neubearbeitete und erweiterte Auflage. Georg Thieme Verlag, Stuttgart New York

van Wezel AP, Opperhuizen A (1995) Narcosis due to environmental pollutants in aquatic organisms: Residue-based toxicity, mechanisms, and membrane burdens. Critical Reviews in Toxicology 25(3):255-279

Verhaar HJM, Leeuwen CJ, Hermens JLM (1992) Classifying environmental pollutants. 1: structure-activity relationships for prediction of aquatic toxicity. Chemosphere 25:471-491

Verslycke T, Roast, SD, Widdows J, Jones MB, Janssen CR (2004) Cellular energy allocation and scope for growth in the estuarine mysid Neomysis integer (Crustacea: Mysidacea) following chlorpyrifos exposure: a method comparison. Journal of Experimental Marine Biology and Ecology 306(1):1-16

Wheeler J, Sorokin N, Leung K, Morritt D, Crane M, Whitehouse P, Cole S, Mitchell R, Holmes D, Rogers HR, Karman C (2001) Marine risk assessment and ecosystem dynamics: comparison of marine and freshwater data and test methods. CO 4972

Wheeler JR, Leung KMY, Morritt D, Sorokin N, Rogers H, Toy R, Holt M, Whitehouse P, Crane M (2002) Freshwater to saltwater toxicity extrapolation using species sensitivity distributions. Environ Toxicol Chem 21:2459-2467 